\section{P03 WHAT DO PEOPLE IN THE GENERAL POPULATION THINK ABOUT BACK PAIN - AND DOES IT MATTER? A SYSTEMATIC REVIEW}

1,2,3,4 L Morton* ${ }^{3,4} \mathrm{M}$ de Bruin, ${ }^{1,2,4} \mathrm{GJ}$ Macfarlane. ${ }^{1}$ Epidemiology Group, University of Aberdeen, Aberdeen, UK; ${ }^{2}$ Aberdeen Centre for Arthritis and Musculoskeletal Health, University of Aberdeen, Aberdeen, UK; ${ }^{3}$ Health Psychology Group, University of Aberdeen, Aberdeen, UK; ${ }^{4}$ Arthritis Research UK/MRC Centre for Musculoskeletal Health and Work, University of Southampton, Southampton, UK

\subsection{6/jech-2017-SSMAbstracts. 105}

Background Mass media campaigns have aimed to change the public's beliefs about back pain to ultimately change behaviours like healthcare utilisation. Most campaigns demonstrated shifts in beliefs without concomitant changes in behaviours. This raises the question of whether the beliefs targeted by campaigns were prevalent and related to the behaviours they tried to change. The objectives of this review were to: i) describe the prevalence of particular beliefs about back pain, ii) identify any cross-sectional relationships between these beliefs and other (e.g. sociodemographic) factors, and iii) assess the evidence for beliefs about back pain to predict future back pain-related outcomes within the general population.

Methods A systematic review of the literature was carried out using searches for 'back pain', 'beliefs' and 'general population' in five literature databases (MEDLINE, PsycINFO, Embase, ISI Web of Science, CINAHL). Articles were screened, data was extracted and quality was assessed by two reviewers. Results across the three aims were tabulated and narratively synthesised.

Results 4480 unique records were identified and 30 articles were included. Beliefs about back pain were measured using eight questionnaire instruments and 57 unique items. These represented beliefs about: consequences, fearing and avoiding pain/activities, catastrophising, and other specific beliefs. Where a given construct was assessed by more than one study, most samples agreed, on average, with beliefs about negative consequences of back pain (8/12), while most disagreed with fear avoidance beliefs about activity (4/5). Negative beliefs about consequences were consistently associated with being older, unemployed, having completed less education, lower self-rated health, and more pain and disability; they were predictive of future back pain-related outcomes.

Discussion Most samples agreed with beliefs about back pain's negative consequences and these beliefs predicted some back pain-related outcomes. However, based on current evidence, we cannot say whether these beliefs impact future management behaviours. The relationships between other belief constructs and future management behaviours has not been investigated in the general population. Previous campaigns' mixed success could, in part, be due to not targeting beliefs which were related to behaviours they hoped to change. Existing evidence suggests that this relationship has not been assessed in the general population; it must be elucidated prior to developing future campaigns.

\section{P04 CHILD-PARENT SEPARATION DURING UPBRINGING AND LATER RISK OF VIOLENT CRIMINALITY AND SELF-HARM: A POPULATION-BASED COHORT STUDY}

${ }^{1} \mathrm{PLH}$ Mok*, ${ }^{2,3} \mathrm{~A}$ Astrup, ${ }^{2,3} \mathrm{CB}$ Pedersen, ${ }^{1} \mathrm{MJ}$ Carr, ${ }^{1} \mathrm{RT}$ Webb. ${ }^{1}$ Centre for Mental Health and Safety, University of Manchester, UK; ${ }^{2}$ Centre for Integrated Register-based Research, CIRRAU, Aarhus University, Aarhus, Denmark; ${ }^{3}$ National Centre for Register-Based Research, Business and Social Sciences, Aarhus University, Aarhus, Denmark

\subsection{6/jech-2017-SSMAbstracts. 106}

Background Childhood experience of parental separation has been associated with subsequent increased risk for interpersonal and self-directed violence in offspring. However, conflicting and insufficient evidence exists regarding how links vary across age, duration, and types of separation. We conducted a large, detailed epidemiological study of the association between child-parent separation and later risk of violent criminality and self-harm in offspring. We investigated how risks varied by type of separation (from mother or father only, or both), offspring gender, age at separation, number and duration of separations, and by a range of separation scenarios.

Methods Our study cohort was established using data from the Danish Civil Registration System and interlinked psychiatric and crime national registers. Persons born in Denmark 1971-1997 $(n=1,346,772) \quad$ were included. Residential addresses were used to determine if cohort members were living with either parent each birthday from birth to 15 th. Members were followed up from reaching age 15 birthday until date of first violent offence conviction, date of first registered self-harm episode, or end of study (31 st December 2012). Incidence rate ratios (IRRs) were estimated using survival analyses techniques in SAS 9.4.

Results All types of child-parent separation scenarios are linked with increased risks for later violent offending and self-harm. Even those who were separated from one parent, but subsequently lived with both, were at increased risk. Compared to persons who always lived with both parents, those who lived with both parents at birth but were subsequently separated from the father-the most common type of separation scenario-had a 2-fold increased risk (IRR for violent offending $=1.99$, 95\% CI 1.94-2.05; IRR for self-harm=2.07, 2.012.14). Risks increased with rising number of parental changes, and a steep gradient in risk with increasing age at separation was observed if children were first separated from both parents between age 10 and 15 years. Strengths of associations were much greater for female than male offspring for violent offending, but no gender differentials in risks were found for self-harm.

Conclusion Our study adds to existing published evidence that experience of child-parent separation may increase the risk for harmful psychosocial development in offspring. However, we had no information on the reasons of separation and aggregated risk estimates would have masked any heterogeneity in the associations observed. Interventions should be tailored toward those young people with the most complex trajectories who have lived through many family changes as they grew up. 